$\begin{array}{cl}\begin{array}{cl}\text { Revue } \\ \text { de Ihistoire }\end{array} & \text { Revue de l'histoire des religions } \\ \text { des religions } & 4 \mid 2010 \\ & \text { Qu'est-ce qu'un « paysage religieux »? }\end{array}$

\title{
Inventaire des temples de Pékin (1750-1949) : épigraphie, archives et enquêtes de terrain
}

A Survey of Peking's Temples (1750-1949): Inscriptions, Archives and Fieldwork

\section{Marianne Bujard}

\section{(2) OpenEdition}

\section{Journals}

Édition électronique

URL : http://journals.openedition.org/rhr/7685

DOI : $10.4000 /$ rhr.7685

ISSN : 2105-2573

Éditeur

Armand Colin

Édition imprimée

Date de publication : 1 décembre 2010

Pagination : 663-682

ISBN : 978-2200-92658-8

ISSN : 0035-1423

Référence électronique

Marianne Bujard, «Inventaire des temples de Pékin (1750-1949) : épigraphie, archives et enquêtes de terrain », Revue de l'histoire des religions [En ligne], 4 | 2010, mis en ligne le 01 décembre 2013, consulté le 03 mai 2019. URL : http://journals.openedition.org/rhr/7685 ; DOI : 10.4000/rhr.7685 


\section{Inventaire des temples de Pékin (1750-1949) : épigraphie, archives et enquêtes de terrain}

Le texte qui suit est une présentation du programme Épigraphie et mémoire orale des temples de Pékin, histoire sociale d'une capitale d'empire, mené en collaboration par l'École française d'Extrême-Orient et l'université normale de Pékin. Son objectif est l'inventaire des temples d'une partie de la ville de Pékin et l'étude de leur histoire et de leur fonctionnement à partir de plusieurs types de sources : l'épigraphie, soit plus de 500 inscriptions conservées sous la forme d'estampages, les archives de l'époque impériale et républicaine sur les biens mobiliers et immobiliers des sanctuaires, leur transmission et leur personnel religieux et les résultats des enquêtes de terrain qui ont permis de localiser les 1100 temples inventoriés à ce jour.

\section{A Survey of Peking's Temples (1750-1949) : Inscriptions, Archives and Fieldwork}

This article presents the data gathered through the project The Temples of Peking : Epigraphy and Oral Sources: The Social History of an Imperial Capital, jointly undertaken by the École française d'Extrême-Orient and Peking Normal University. The program aims at making a complete survey of the temples of the inner city of Peking and at studying their history and social functions. The main sources are: over five hundred inscriptions preserved in rubbings; documentation found in imperial and republican archives, which provide information on the real estate and moveable property owned by the religious foundations and their clerics; and the results of interviews and fieldwork, which made it possible to localize the 1100 temples surveyed to this day. 
Le texte qui suit est une présentation du programme Épigraphie et mémoire orale des temples de Pékin, histoire sociale d'une capitale d'empire, conduit en collaboration par l'École française d'Extrême-orient et l'université normale de Pékin.

L'idée d'entreprendre un inventaire des temples de Pékin n'est pas née d'une pure curiosité intellectuelle mais s'est imposée à nous à la vue des destructions accélérées que subissaient, depuis les années 1990, les vieux quartiers de Pékin. En cherchant à recueillir les témoignages des anciens habitants sur les activités religieuses qui se développaient autour des temples avant la révolution de 1949, nous nous inscrivions, sans le savoir alors, dans une tradition indigène qui remontait au moins $\mathrm{au} \mathrm{XVI} \mathrm{e}^{\mathrm{e}}$ siècle, lorsque furent écrits les premiers ouvrages consacrés aux monuments de la capitale et aux coutumes de ses habitants. Tout au long de l'empire, lettrés et amateurs cultivés avaient recensé les sites remarquables et les habitudes locales dans le but d'en préserver la mémoire et d'en faire partager le goût aux visiteurs. Par la suite, la disparition du régime impérial et l'instabilité politique des premières décennies du $\mathrm{XX}^{\mathrm{e}}$ siècle renforcèrent l'attrait pour les choses anciennes, donnant lieu à toute une littérature dédiée à l'histoire de Pékin, mais aussi à des campagnes d'inventaire des monuments, à des enquêtes sur les croyances et les modes de vie de ses habitants, conscients d'être les héritiers d'une culture urbaine unique. Dans l'identité que la ville s'était construite au cours des âges, les temples avaient toujours occupé une place privilégiée.

Notre principale hypothèse de départ s'inspirait des travaux de Kristofer Schipper au sujet des fonctions socioreligieuses ou « liturgiques » des temples de quartier, telles qu'il les avait observées à Tainan, au sud de l'île de Taiwan ${ }^{1}$. Pour lui, en dehors des actes de dévotion, les associations de fidèles qui en assuraient l'entretien jouaient un rôle beaucoup plus large, car l'activité religieuse comprenait aussi des actions séculières de bien public, comme l'aide aux

1. Kristofer Schipper, «Neighborhood Cult Association in Traditional Taiwan », in William Skinner (éd.), The City in Late Imperial China, Stanford, 1977, p. 651-676. 
déshérités et l'éducation, mais aussi l'entretien des rues, la sécurité des quartiers, la protection contre les incendies ou toute autre tâche utile aux habitants. Poursuivant dans ce sens, on pouvait montrer que la gestion de la cité reposait aussi sur des regroupements autonomes d'individus liés par la foi autour d'un réseau de temples, et qu'elle échappait, pour une part du moins, aux édiles de l'empire, et plus tard à ceux du régime républicain. L'enjeu était d'éclairer les contours d'une « société civile » coexistant avec les institutions impériales, puis républicaines.

Le nombre élevé de temples dans la capitale semblait militer en faveur de cette hypothèse. Les données recueillies par Susan Naquin, l'éminente spécialiste de l'histoire des fondations religieuses de Pékin, fondées sur l'épigraphie et la littérature, font état, entre 1403 et 1911, de 2564 temples dans la ville intra-muros et ses proches environs. À ce nombre solidement établi par les sources, 303 autres, moins bien documentés, peuvent encore s'ajouter ${ }^{2}$. Susan Naquin estime que le total ainsi obtenu est probablement en dessous de la réalité. Un registre manuscrit d'enregistrement des temples, composé durant le règne de l'empereur Qianlong (1735-1796), le confirme : le nombre des seuls temples occupés par des moines ou des nonnes bouddhistes se monte à $2240^{3}$. À ce chiffre, il faudrait encore ajouter les fondations lamaïstes, taoïstes et les petits sanctuaires sans religieux à demeure. On peut donc avancer le chiffre de 3000 temples sans risque d'exagération, soit pour une population qui se situait à partir du $\mathrm{XIX}^{\mathrm{e}}$ siècle (en incluant les faubourgs) entre 800000 et un million d'habitants, près d'un temple pour trois cents personnes.

Afin de mieux appréhender le rôle des fondations religieuses dans l'espace urbain, nous avons choisi de recueillir une série de matériaux permettant d'en reconstituer l'histoire et le fonctionnement. Notre enquête se limite à la partie nord de la ville, que l'on appelle la «ville intérieure »; mieux préservée, celle-ci devait en toute hypothèse non seulement renfermer des constructions anciennes,

2. Susan Naquin, Peking-Temples and City Life, 1400-1900, Berkeley, 2000.

3. Bacheng miaoyu sengni zongce, compilé à la fin de l'ère Qianlong, après 1769 , et conservé à la Bibliothèque de l'Institut d'histoire de l'Académie des sciences sociales de Chine à Pékin. 
mais aussi abriter des résidents établis de longue date. Ces derniers pouvaient éventuellement témoigner des activités cultuelles d'avant 1949, année de fondation de la République populaire de Chine à partir de laquelle l'exercice de la religion fut fortement limité, puis interdit.

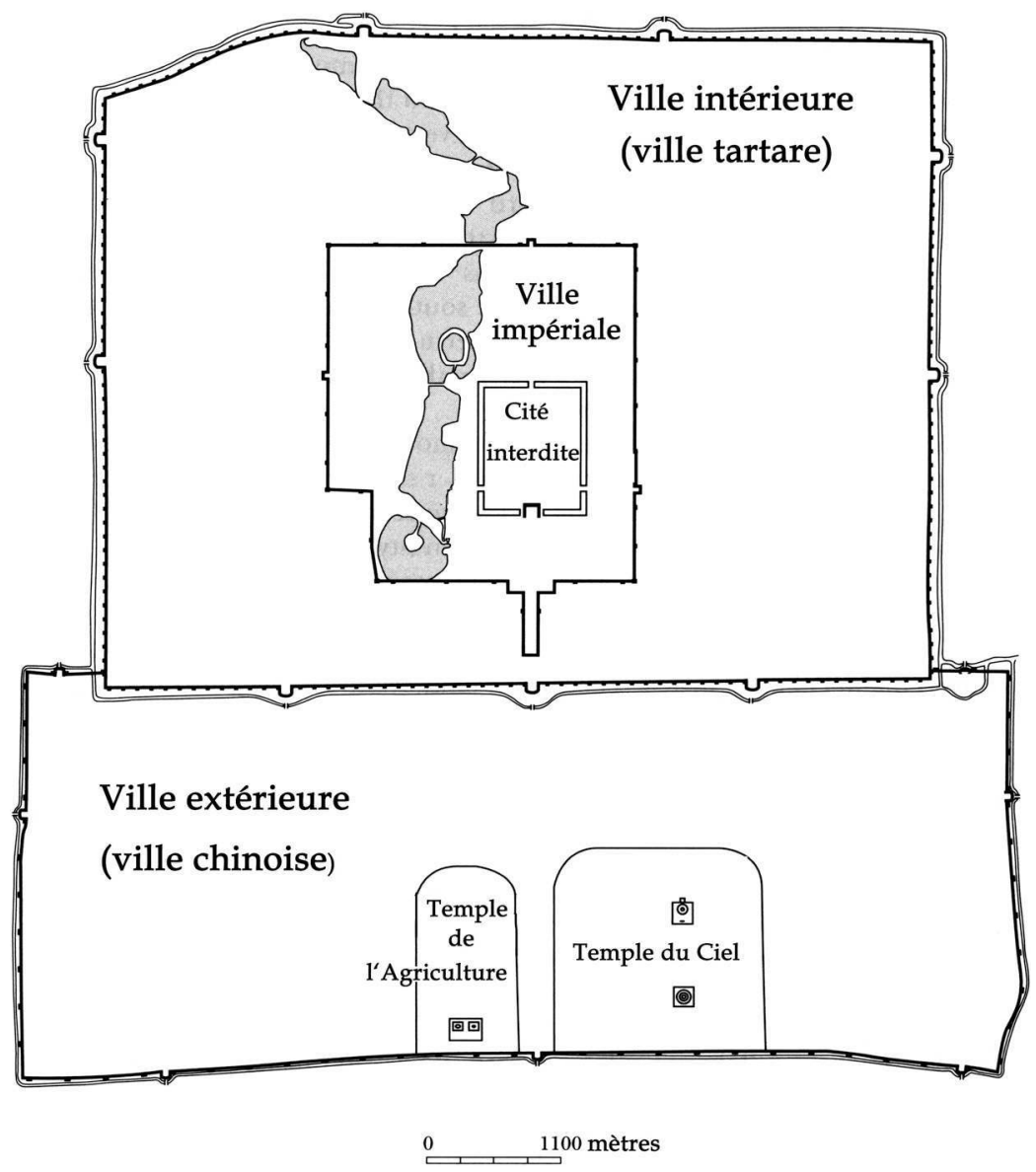

Pékin intra-muros : le périmètre de la ville intérieure 
La première étape du travail a consisté à dresser un inventaire des temples de la ville intérieure en partant d'une ancienne carte de Pékin, dessinée en 1750, qui reproduit maison par maison, y compris les étages, toutes les constructions de la ville de Pékin intra-muros. Le savant Yang Naiji a démontré que cette carte, commandée par l'empereur Qianlong, reproduit avec une grande fidélité l'état du bâti de l'époque ${ }^{4}$. La ville y était représentée sur dix-sept bandes parallèles alignées du nord au sud, chaque bande étant elle-même divisée en trois sections - est, centre, ouest - pliées séparément les unes des autres et formant au total cinquante-et-un cahiers. Les dimensions de cette gigantesque carte étaient de 13,504 $\mathrm{m}$ de large sur 14,144 de long, soit une représentation de Pékin au $1: 650^{\mathrm{e}}$. À la fin du XIX ${ }^{\mathrm{e}}$ siècle, une copie en fut réalisée à la même échelle tout en faisant apparaître certaines nouvelles dispositions des administrations et des palais. En 1940, deux reproductions de la carte de Qianlong furent publiées à une échelle plus petite. La première au 1:2400 , réalisée par le Musée du Palais, prend la forme de feuilles séparées réunies dans un coffret - chacune des dix-sept bandes étant reproduites sur douze feuilles volantes, ce qui représente un total de deux cent quatre feuilles, dont trois sont vierges (il s'agit de portions hors les murs). L'autre copie fut entreprise par un organisme dépendant des forces japonaises d'occupation, le Bureau des affaires politiques du département de liaison de la Chine du Nord (Xingyayuan Huabei lianluobu zhengwuju). Elle reprend la disposition d'origine en cinquante-et-un cahiers, mais au 1:2600 ${ }^{\mathrm{e}}$. Enfin, en 1995 parut une nouvelle édition sous la forme de feuilles séparées reproduisant à main levée l'édition du Musée du Palais.

Pour repérer les temples, nous nous sommes servis des trois copies à notre disposition. En complément, nous avons utilisé plusieurs cartes de l'époque républicaine et une carte de la première année de la République populaire. Bien qu'aucun de ces derniers documents ne puisse être comparé - en précision et par le nombre de temples représentés - avec la carte de 1750, ils nous ont permis à la fois de situer les temples anciens et de repérer les temples nouveaux, construits après 1750 .

4. Yang Naiji, « Qianlong Jingcheng quantu kaolüe », Gugong bowuyuankan 3 (1984), p. 7-24. 
La ville intérieure occupe onze des dix-sept bandes de la carte de 1750; chaque bande est divisée en douze sections, formant chacune un « îlot» ou paiduan. Les onze îlots situés à l'extérieur des remparts de l'ouest, ainsi que celui du coin nord-ouest de la ville étant vides, le total des feuilles documentées pour la ville nord se monte à cent vingt. Sur ces cent vingt îlots, apparaissent huit cent vingt-neuf temples dont les noms sont inscrits et les bâtiments dessinés. La présence d'environ trois cents temples supplémentaires est attestée par diverses autres sources : les cartes plus récentes, l'épigraphie, la littérature, les documents tels que les archives, les registres du clergé bouddhiste, les inventaires tardifs des temples et les données recueillies par les enquêtes. Le nombre des temples de la ville intérieure attestés entre 1750 et 1949 est donc d'environ onze cents.

\section{ÉPIGRAPHIE}

Pour étudier ces temples, on dispose de plusieurs sources, la littérature historique, très abondante sur Pékin, les archives, celles de l'empire mandchou (1644-1911) et celles de la République (1911-1949), et les inscriptions des stèles érigées dans les temples. Ces derniers n'abritaient pas tous des stèles et nous disposons pour la ville intérieure de cinq cent quarante et une inscriptions relatives à deux cent vingt et un temples, soit moins d'un quart des quelque mille cent fondations religieuses inventoriées. Rédigées entre le $\mathrm{XIII}^{\mathrm{e}}$ et le milieu du $\mathrm{XX}^{\mathrm{e}}$ siècle, ces inscriptions couvrent plus de sept cents ans d'histoire et apportent une documentation de première main. On les étudie à partir des estampages réalisés dans la première moitié $\mathrm{du} \mathrm{Xx}^{\mathrm{e}}$ siècle, car la plupart des stèles ont disparu, certaines sont encore enterrées, d'autres encastrées dans les parois ${ }^{5}$.

L'épigraphie permet de reconstituer en partie l'histoire des temples, d'identifier les dieux que l'on y priait, de connaître les noms des religieux qui célébraient les cultes, et ceux des personnages qui fondèrent ou restaurèrent les sanctuaires. La disposition

5. Une grande partie des estampages sont reproduits dans la collection en cent volumes Beijing tushuguan cang Zhongguo lidai shike taben huibian, Beijing tushuguan jinshizu (éd.), Zhengzhou, Zhongguo guji, 1990-1991 [dorénavant $B J T B]$. 
générale des lieux est quelquefois décrite et, au verso des stèles, se trouvent souvent les noms des donateurs qui, parfois par centaines, financèrent les réparations des bâtiments. Les rédacteurs des inscriptions ne sont pas toujours connus. Dans notre corpus, seule la moitié des textes gravés est signée. Les rédacteurs se répartissent en trois groupes principaux : les empereurs, les lettrés fonctionnaires et les religieux. Les empereurs sont les auteurs de plus d'un quart des inscriptions signées. Les stèles impériales célèbrent des fondations, des cérémonies d'actions de grâces rendues à une divinité particulière, soit dans un temple entretenu par l'administration impériale, soit dans un sanctuaire ouvert au public que l'empereur veut honorer d'une attention particulière. Les stèles qui font état de donations de terrains ou de textes sacrés, celles qui décernent des titres honorifiques à des héros ou à des religieux sont le plus souvent de la main de l'empereur. Les lettrés fonctionnaires, surtout ceux de l'Académie Hanlin, du Collège impérial mais aussi du ministère des Rites ou d'autres administrations, sont les auteurs de plus de la moitié des stèles signées de la ville intérieure (155 sur 278). Soit ils rédigent des textes en rapport direct avec les cultes dont ils ont eu l'initiative (ou la responsabilité dans le cas des sacrifices officiels), soit ils sont invités par des communautés de fidèles, des religieux ou des eunuques à composer une inscription pour un temple avec lequel ils n'entretiennent pas nécessairement de rapport direct. Cependant, avant d'évoquer dans ce cas une simple commande, il faut examiner les circonstances qui ont amené à recourir à un lettré. Parfois, le rédacteur éclaire le lien qui l'a rapproché du temple : son amitié avec un religieux, un séjour sur place en tant que locataire. Les religieux ont aussi composé des inscriptions, mais cellesci représentent moins d'un dixième du corpus. Elles sont le plus souvent de la main du régisseur du temple (zhuchi) qui y consigne les transferts de propriété ou les réparations. Des moines éminents rédigent également des inscriptions parce que des liens personnels les attachent à un lieu de culte, ou que celui-ci est une dépendance (xiayuan) du monastère qu'ils habitent.

Dans la grande majorité des cas, les commanditaires de la stèle célèbrent la restauration du sanctuaire ou de l'une de ses salles, parfois ils tiennent aussi à rappeler, en le consignant dans la pierre, qu'une cérémonie s'est tenue pour rendre grâce d'un bienfait particulier. Ainsi, dans un temple de Guandi, à l'ouest de 
Dongzhimen, une inscription célèbre la générosité d'un bienfaiteur qui s'est chargé de la reconstruction complète du sanctuaire, meubles et ustensiles compris ${ }^{6}$. D'autres inscriptions, à l'occasion d'une réfection, fixent les limites exactes du périmètre occupé par le temple et ses dépendances dans le but évident d'en protéger la propriété ${ }^{7}$.

Certaines stèles proclament des règlements relatifs au fonctionnement d'une association ou d'une guilde professionnelle liée à un temple : dans le Zhenwumiao de Xisibeidajie, une stèle énumère les obligations des membres de la guilde des courtiers de viande de porc à l'égard du dieu des Richesses; parmi celles-ci, le paiement d'une taxe sur chaque animal vendu, la représentation d'un opéra et l'organisation d'un banquet pour l'ouverture d'un nouveau commerce ${ }^{8}$.

D'autres inscriptions définissent la propriété des temples, par exemple en interdisant la transformation d'un temple "public» appartenant au clergé (shifang congling) en temple héréditaire (zisunmiao), dont la transmission de maître à disciple priverait la communauté monastique de ses droits ${ }^{9}$. Les inscriptions peuvent encore constituer des contrats enregistrant l'achat de terrains ou la donation d'une terre par un particulier. Dans une stèle érigée en 1808 dans le Wanfosi de Xiaoheihu hutong, un Mandchou rembourse ainsi une dette contractée auprès du révérend du temple en cédant deux terrains des environs de Pékin. L'inscription note alors l'emplacement des terrains, leur cession inaliénable et les noms des témoins qui garantissent la transaction ${ }^{10}$.

Une stèle reproduit parfois un sutra ou un verset de sutra, accompagné dans certains cas de l'image gravée d'une divinité ${ }^{11}$. La stèle n'est alors plus seulement commémorative, mais constitue au même titre qu'une statue, un objet de dévotion.

Comme on le voit, le contenu des stèles varie, mais toutes possèdent une valeur contractuelle qui engage aussi bien les dieux envers les hommes que les hommes entre eux. D'un côté, il s'agit

6. BJTB, \# 200 (1833), vol. 80, p. 48.

7. BJTB, \# 8420 (non datée), vol. 60 , p. 185 , pour le Huguosi.

8. BJTB, \# 1091 (1849), vol. 81, p. 181-182.

9. BJTB, \# 518 (1673), vol. 63 , p. 35.

10. BJTB, \# 466 (1808), vol. 78, p. 34.

11. BJTB, \# 367 (1592), vol. 58, p. 28. 
de faire connaître aux divinités des lieux les circonstances qui ont présidé à la restauration du sanctuaire et l'identité des contributeurs; ceux-ci espèrent que les dieux les favoriseront en retour, tandis que leurs noms gravés dans la pierre pérennisent leur présence dans le temple. De l'autre, la stèle est un contrat passé entre les hommes. Lorsque son texte précise les dimensions du temple, mentionne un don ou un achat de terrain, sa valeur juridique est évidente; c'est encore le cas dans les inscriptions qui détaillent les règles d'une guilde ou d'une association. Lorsqu'il s'agit d'une stèle commémorant la restauration d'un bâtiment, tous ceux dont les noms sont inscrits, en commençant par les plus généreux donateurs, peuvent s'attendre à jouir d'un prestige accru au sein de la communauté des fidèles. Lorsqu'une association de fidèles revendique, stèles à l'appui, une activité continue durant plusieurs décennies dans un sanctuaire particulier, elle est en mesure de faire valoir son droit à y poursuivre ses dévotions. C'est le cas de l'association de bienfaisance, active pendant près de trente ans dans le grand temple du roi des Médicaments (Bei Yaowangmiao) situé dans le nord de la ville. La répétition des noms des mêmes donateurs d'une stèle à l'autre y démontre en outre la stabilité des effectifs de l'association $^{12}$.

Une mention particulière doit être réservée aux eunuques qui fondèrent et entretinrent plusieurs centaines de temples dans Pékin et ses environs (140 fondations et 294 temples subventionnés). Dans la ville intérieure, ils figurent au titre de donateurs dans quatrevingt-treize inscriptions relatives à cinquante-cinq temples. C'est surtout durant la dynastie des Ming, lorsqu'ils étaient au sommet de leur puissance, que leur patronage est le plus manifeste. Il est alors fréquent qu'un eunuque transforme sa résidence privée en temple; il y nomme un prêtre et s'y retire au moment où il quitte le service de la cour. C'est le cas du Qingxuguan, dont il ne reste que la stèle de fondation. Datée de 1454, elle gît abandonnée au milieu d'un potager, non loin de la tour de la Cloche ${ }^{13}$. Tandis que soixante-deux stèles des Ming commémorent les donations diverses d'eunuques, vingt-six seulement signalent leur patronage durant la

12. BJTB, \# 494 (1752), vol. 70, p. 176-177; BJTB, \# 545 (1779), vol. 74, p. $33-34$.

13. BJTB, \# 550 (1454), vol. 51, p. 190. 
période mandchoue, au cours de laquelle leur influence fut fortement réduite par la volonté des empereurs. Sur ces vingt-six inscriptions, dix-sept sont postérieures au milieu du $\mathrm{XIX}^{\mathrm{e}}$ siècle, montrant un regain de leur activité. Pour la dynastie des Qing, on possède plusieurs inscriptions relatant l'achat en commun par des eunuques d'un temple dans lequel ils prévoient de s'installer au moment de la retraite, comme celle où, en accord avec les moines du Xinglongsi, situés au nord-ouest de la Cité interdite, des eunuques constituent en 1761 un fonds de retraite pour assurer leurs vieux jours et résider dans le temple ${ }^{14}$.

Dans notre corpus, les commerçants n'apparaissent explicitement comme donateurs qu'après 1750. Dans certains cas, leurs boutiques sont situées à proximité du temple qu'ils subventionnent, mais elles peuvent aussi être dispersées dans la capitale, voire être établies dans une autre ville. L'une des premières stèles date de 1753 et mentionne trente noms de magasins dans la liste des donateurs. Son érection dans le Wushengmiao, situé à Xinjiekou, fut suivie par celle de trois autres stèles qui, toutes, comportent des noms de commerces. Plusieurs noms se retrouvent d'une stèle à l'autre. Cette continuité remarquable montre que les regroupements de fidèles n'ont pas été seulement des phénomènes éphémères, sans stabilité, ni longévité ${ }^{15}$.

Nous comptons soixante-huit stèles qui font état d'environ soixante associations. Pour autant qu'il soit possible de le déterminer, ces associations se divisent en associations occasionnelles - formées pour la réparation d'un temple, la célébration d'une divinité -, et en associations durables. Ces dernières peuvent regrouper des hommes exerçant des fonctions administratives identiques, comme les censeurs (jiancha yushi) qui sacrifiaient tous les ans à leur propre dieu du Sol dans un temple de Guandi au sud de la Cité interdite. De telles associations existaient chez les fonctionnaires ou les eunuques qui géraient les greniers, les armureries, les poudrières, les écuries, les filatures. Vingt-deux guildes participaient à l'entretien des temples : celles des charpentiers, des menuisiers, des porteurs d'eau, des changeurs, des vendeurs de fruits frais et secs, des

14. BJTB, \# 674 (1761), vol. 71, p. 195 ; association toujours active en 1895 , voir $B J T B$, \# 678, vol. 87, p. 115.

15. BJTB, \# 372 (1753), vol. 71, p. 19; BJTB, \# 374 (1774), vol. 71, p. 19-20; BJTB, \#371 (1794), vol. 76, p. 85-86; BJTB, \#370 (1821), vol. 79, p. 8-9. 
fripiers, des marchands de soie, des conteurs. La plupart regroupait des individus d'un quartier, résidant près d'un marché, ou près d'un puits, dans le cas des porteurs d'eau. On a identifié une trentaine d'associations formées autour d'un culte dont les membres n'avaient en commun que la volonté de se vouer au service d'une divinité particulière. Par exemple, l'association des Pénitents du temple du dieu de la Cité de la Capitale, situé au sud-ouest de la ville, qui marchait en tête de la procession du dieu pour prêcher la vertu ${ }^{16}$; ou celle des fidèles, toutes des femmes, qui célébraient le culte de la Bonne Mère des Neuf Sommets (Jiuding niangniangmiao) dans un temple dédié à cette divinité et situé derrière la tour du Tambour, à côté de celui du roi des Médicaments ${ }^{17}$.

En dépit du fait que les sources épigraphiques n'éclairent que des moments de l'histoire d'un temple, comme les réparations, les dons de terrains et d'objets ou l'installation d'un moine célèbre, elles représentent souvent la seule documentation permettant de retracer l'histoire des sanctuaires. Produites par les acteurs des cultes euxmêmes, elles procurent surtout un témoignage direct sur la vie religieuse de la capitale.

\section{Les ARChIVes des QING}

L'épigraphie nous éclaire sur le patronage impérial que recevaient les temples puisqu'un quart des inscriptions «signées» émane de la main des empereurs. Mais pour saisir l'ampleur du soutien accordé par la cour aux temples et aux cultes des divinités en général, il faut se plonger dans la masse des archives de l'empire mandchou.

Plusieurs ministères étaient impliqués à des degrés divers dans la gestion des temples et du clergé. Le ministère des Travaux publics intervenait dans les constructions et les réparations, le ministère des Finances dans le contrôle et la vente des certificats d'ordination, le ministère de la Justice pour les affaires criminelles qui touchaient le clergé. Mais trois organismes, hérités de la dynastie des Ming, administraient plus directement les fondations religieuses et leurs offi- 
ciants, sans exercer pour autant un contrôle strict et régulier ni sur le fonctionnement des lieux de cultes ni sur l'orthodoxie des dogmes. Il s'agissait du Bureau d'enregistrement des moines bouddhistes, le Senglusi, puis de son équivalent pour le clergé taoïste, le Daolusi, et pour les fondations lamaïstes, le Lama yinwuchu. Les deux premiers appartenaient au ministère des Rites et le troisième était rattaché à la cour des Affaires coloniales (Lifanyuan). Dans le cas des fondations taoïstes, le clergé du Longhushan, siège de l'Église des maîtres célestes au Jiangxi, avait aussi ses représentants à Pékin et dans le reste de l'empire, ceux-ci occupaient parfois les postes du Daolusi ${ }^{18}$.

Dans la masse des archives de la dynastie des Qing, il existe des milliers de documents utiles pour évaluer de façon très précise l'investissement consenti par l'État et la cour à l'entretien des temples et du clergé. Malheureusement, leur dépouillement complet n'a pas été rendu possible dans le cadre de ce programme et nous n'avons utilisé qu'un nombre limité de matériaux. Ceux-ci proviennent essentiellement du fonds d'archives de la Maison impériale ${ }^{19}$, car c'est avant tout par le biais de cette administration que l'empereur accordait son soutien à plus d'une centaine de temples dont près d'un tiers se trouvait dans la ville intérieure. L'index informatisé du fonds des mémoires du Palais, rédigé par les services administratifs de la Maison impériale, dénombre 91483 documents (cote 05) couvrant pratiquement l'ensemble de la dynastie. Une première évaluation indique qu'à partir du règne de Qianlong, quelque six mille d'entre eux concernent les temples de la capitale. Il s'agit de requêtes adressées principalement par les responsables du bureau des Cérémonies, souvent par l'un des grands intendants de la Maison impériale, parfois par le bureau des Constructions pour les réparations ou, pour les distributions d'objets liturgiques, par le Bureau des magasins. La très grande majorité des requêtes concerne les cérémonies qui se tenaient à l'occasion des fêtes du Nouvel An, des anniversaires de l'empereur et de l'impératrice douairière, et

18. Voir Vincent Goossaert, The Taoists of Peking 1800 - 1949, A Social History of Urban Clerics, Cambridge - Londres, 2007, p. 59-62.

19. Administration comportant de nombreux bureaux fonctionnant sur les ressources propres de l'empereur et dédiée à la gestion des biens privés du souverain et de ses proches parents. 
de l'anniversaire du Bouddha. Plus d'un dixième (673 dossiers) se rapportent au Hongrensi ${ }^{20}$, fondation surtout active sous Qianlong, où les lamas accomplissaient tout au long de l'année des rituels qui les réunissaient par centaines pour la récitation des sutras, tandis que les acteurs au service de la cour venaient y jouer de l'opéra. Des cérémonies comparables se déroulaient dans les temples de la Cité Interdite, dans les sanctuaires des collines de l'ouest (Xishan) et des parcs impériaux situés en ville et dans le temple des Lamas (Yonghegong). Elles duraient dix jours et réunissaient des centaines d'officiants ${ }^{21}$. Un document daté de la $35^{\mathrm{e}}$ année de l'ère Qianlong (1770) nous renseigne sur l'emplacement de soixante-douze temples subventionnés (guanguan simiao) ${ }^{22}$. Parmi eux, seulement cinq étaient dans la ville sud, tandis que plus de la moitié (40) s'élevaient dans la ville intérieure.

Le soutien de la cour se manifestait par des réparations entreprises aux frais de la cassette impériale, et des dons en nature étaient régulièrement accordés à plusieurs dizaines de lieux de cultes : bouddhas en terre cuite, rouleaux de peintures, écharpes et pièces de soie, sachets d'encens, lampes ou ustensiles d'autel. D'autres documents font état de subsides réguliers versés pour l'achat d'encens à une vingtaine de temples durant le règne de Qianlong. On possède aussi le décompte des subsides alloués à près de quatre-vingts temples durant la troisième année de l'ère Jiaqing (1798) pour l'encens, les bougies, la nourriture des moines et l'entretien des novices : au total, 949,5 taëls ${ }^{23}$.

Ces quelques données recueillies dans les archives de la dynastie mandchoue révèlent l'existence d'une véritable économie religieuse dans laquelle des sommes colossales étaient allouées régulièrement aux services religieux et à l'entretien des temples. Ainsi, pas moins d'un million de taëls fut dépensé par l'empereur

20. Ce temple était durant les Ming et au début des Qing une chapelle fudian que Kangxi agrandit pour y installer une précieuse statue du Bouddha en bois de santal. Saccagé et incendié en 1860, on construisit sur son site des casernes pour les troupes affectées à la protection de Pékin; aujourd'hui le site est occupé par l'armée et n'est pas accessible.

21. Archives $n^{\circ} 1$, dossier 05-0291-051.

22. Archives $n^{\circ} 1$, dossier 05-0277-032.

23. Archives $n^{\circ} 1$, Neiwufu Zhangyisi, liasse $11,3^{\mathrm{e}}$ année de l'ère Jiaqing (1798). 
Qianlong au cours de son règne pour la restauration et l'entretien du temple des Lamas ${ }^{24}$.

Un travail plus poussé sera nécessaire pour mieux définir ce que recoupait la notion de " temples subventionnés » (guanguan simiao). Dans la liste des soixante-douze fondations désignées comme telles dans la requête 1770 mentionnée plus haut, la majorité (43) était administrée par le clergé bouddhiste, seize par le clergé taoïste, les autres par des « maîtres de musique et de danse » yuewusheng, et des prêtres taoïstes de l'école Zhengyi affectés au Bureau impérial de la musique pour officier dans les sacrifices impériaux. Les temples subventionnés ne formaient pas un ensemble homogène, ni en terme d'obédience, ni en terme d'ouverture au public. Les subventions accordées par la cour ne les empêchaient pas de louer des salles (douze le faisaient), des grandes fondations mais aussi de modestes temples, comme celui du dieu des Richesses de Gulou dongdajie qui ne disposait que de quatre salles autour d'une cour. Dans une étude récente, Yang Jian oppose les temples officiels et non officiels (guanfang/feiguanfang siyuan). Les premiers étaient tenus de célébrer les cultes et les sacrifices officiels, les anniversaires et les fêtes du calendrier, et recevaient pour cela des subsides en nature et en espèce; leur réparation était financée par la Maison impériale. Les seconds, beaucoup plus nombreux, vivaient de leurs propres revenus et étaient davantage ouverts à la population ${ }^{25}$. En définitive, il faut garder à l'esprit que le patronage impérial autant que les subsides de l'État n'étaient pas accordés une fois pour toutes, et que la liste des temples entretenus variait au cours du temps.

\section{LES ARCHIVES RÉPUBLICAINES}

Afin de documenter l'histoire des temples de notre périmètre au $\mathrm{Xx}^{\mathrm{e}}$ siècle, nous avons dépouillé, de façon plus systématique cette fois, un fonds d'archives constitué par les documents issus des cam-

24. Lai Hui-min, Chang Chu-ya, "Qing Qianlong shiqi de Yonghegong, ge'an jingji wenhua cengmian de guancha », Gugong xueshu yuekan 23-4 (2006), p. 158.

25. Yang Jian, Qing wangchao fojiao shiwu guanli, Pékin, Shehui kexueyuan wenxian chubanshe, 2008, p. 315-321. 
pagnes d'enregistrement des temples, conduites successivement par le bureau de la Sûreté de la ville de Pékin entre 1928 et $1929^{26}$, le bureau des Affaires sociales entre 1930 et $1945^{27}$, et le bureau des Affaires civiles entre 1945-194728. Un millier de dossiers se rapportent à quatre cent cinquante temples parmi les onze cents que nous avons inventoriés.

De façon générale, les archives républicaines ne fournissent aucune information concernant les communautés de fidèles qui fréquentaient les temples ou les cérémonies qui s'y déroulaient. Tout au plus les inventaires détaillent-ils les statues des dieux honorés et les sutras conservés - qui sont peut-être ceux que les religieux récitaient le plus souvent. En réalité, ce qui intéresse les enquêteurs, c'est tout ce qui a trait aux biens du temple : sa valeur mobilière et immobilière, ses terrains, ses revenus, ses garants, ses activités de charité s'il y a lieu, son personnel religieux, les droits de propriété et de succession.

Les efforts déployés pour tenter d'établir les droits de propriété sur les édifices religieux répondaient à une situation créée par les réformes dites des «Cent jours » (1898), puis pendant la décennie des « Nouvelles Politiques » (Xinzheng, 1901-1911) visant à la modernisation de l'appareil d'État et du fonctionnement des institutions. Dans l'effort entrepris pour restructurer le système d'éducation en fondant des écoles publiques, on procéda à la confiscation des temples ou d'une partie de leurs locaux pour y aménager des salles de classe. Les abus furent nombreux, surtout en province où les pouvoirs locaux - publics ou privés - s'autorisèrent à confisquer les biens du clergé, récupérant terrains et bâtiments à leur propre profit sans forcément y créer des écoles, mais en y installant des entreprises privées ou des administrations. Les réactions dispersées du clergé furent d'abord sans cohésion ni efficacité, puis, alors que de nombreuses associations des clergés bouddhiste et taoïste avaient été fondées dans l'empire finissant, celles-ci se virent peu à peu confier par les nouvelles autorités républicaines un rôle d'intermédiaire entre les religieux et l'administration.

26. Les dossiers regroupés sous la cote J181-15- ne sont plus accessibles depuis la fin de l'année 2005.

27. Dossiers regroupés sous la cote J2-8-.

28. Dossiers regroupés sous la cote J3-. 
De fait, les campagnes d'enregistrement des temples visaient d'une part à percevoir des impôts sur leurs biens mobiliers et immobiliers, d'autre part à protéger leur propriété contre les divers prédateurs. Les archives républicaines nous renseignent donc sur plusieurs centaines de temples de petite taille, certains occupés par des moines qui s'en transmettaient non pas la propriété, mais le droit d'y résider et d'y célébrer les cultes, d'autres - des temples privés -, appartenant à une famille ou à un particulier qui en avait l'entière jouissance, et pouvait le vendre ou l'hypothéquer à sa guise.

Si l'activité des fidèles n'est pas visible dans les documents d'archives, les informations relatives à l'identité des religieux, leur affiliation à une école, leur nombre, les relations qu'ils entretiennent avec d'autres temples - souvent un moine est responsable de deux, voire de trois temples - sont abondantes et éclairent l'identité du temple du point de vue du clergé ou des laïcs qui s'en occupaient. En outre, des listes nominatives nous renseignent sur les eunuques qui résidaient dans les temples qu'ils avaient acquis.

\section{LES ENQUÊTES DE TERRAIN}

En même temps que nous recherchions sur le terrain la trace des lieux de culte, nous nous sommes efforcés de recueillir les témoignages des riverains, souvent les habitants des temples désaffectés, sur les activités religieuses passées. C'est grâce à ces enquêtes que nous avons pu retrouver la trace de petits temples de quartier, en général des oratoires dédiés au dieu du Sol, qui n'étaient pas connus par ailleurs, ni par les cartes ou l'épigraphie, ni par la littérature ou les archives. Souvent sans religieux à demeure, ces lieux servaient aux dévotions ordinaires des habitants d'une rue.

Les repérages sur le terrain nous ont aussi renseignés sur la disparition des temples de Pékin. Sur les onze cents temples situés dans le périmètre de la ville intérieure entre 1750 et 1949, il n'en reste aujourd'hui qu'environ deux cents, dont six seulement en activité les autres ayant été transformés en école ou en musée, et le plus souvent reconvertis en habitation ordinaire (de certains ne subsiste plus qu'une salle ou une porte).

La disparition des temples de Pékin s'est déroulée en plusieurs étapes. Dès la fin du XIX ${ }^{\mathrm{e}}$ siècle, leur nombre diminua lors des cam- 
pagnes anticléricales, en même temps que s'appauvrissait la capitale. En 1851, un recensement conduit par la gendarmerie dans la ville intérieure dénombrait huit cent soixante-six temples. En 1908, le ministère des Affaires civiles en recensait encore sept cent deux $^{29}$. Dans les années 1930, l'inventaire des bâtiments des fondations religieuses, effectué entre 1931 et 1933 par l'Institut des arts de Peiping (Beiping yishu yanjiuyuan), comprenait cinq cent soixante-quinze temples abritant ou non des religieux. En 1950, une liste établie par le bureau des Affaires civiles n'en comptait plus qu'environ cinq cents.

Avec l'avènement de la République populaire, les activités religieuses furent fortement ralenties, les formations sectaires interdites. Près de quatre-vingt-dix pour cent du clergé retourna à l'état laïc. Il ressort cependant des enquêtes que, jusqu'au déclenchement de la Révolution culturelle en 1966, on pouvait encore faire appel à des moines pour accomplir les rites funéraires à domicile et que des cultes se maintenaient dans quelques temples dont les statues étaient rassemblées dans la salle principale, qui n'était généralement pas louée.

La situation changea radicalement avec le mouvement de « destruction des quatre vieilleries », lancé le $1^{\text {er }}$ juin 1966. Au cours de l'été, les temples furent saccagés, les statues détruites, les stèles brisées. Les religieux qui n'étaient pas encore retournés à l'état laïc furent regroupés et rééduqués dans quelques monastères. Bien que les édifices fussent alors affectés à d'autres usages, les bâtiments, même dégradés, subsistèrent dans leur majorité jusqu'à la fin des années 1980.

À partir des années 1990, la disparition des temples ne fut pas le résultat d'une politique anti-religieuse, mais bien la conséquence de l'essor immobilier que connut la capitale. Ils disparurent en même temps que les maisons sur cour qui formaient les quartiers anciens. La préservation matérielle de certains d'entre eux jusqu'à un passé relativement récent explique que les habitants s'en souviennent encore. En effet, bien que les dévotions et les cultes aient cessé depuis plus de cinquante ans, les édifices ont longtemps conservé la mémoire des lieux. 


\section{Conclusion}

Revenant à notre hypothèse de départ, il faut bien reconnaître que les matériaux recueillis ne correspondent pas à notre attente. D'une part, le trop grand éloignement dans le temps des faits recherchés, conjugué à une forme de refoulement, sinon d'amnésie concernant les pratiques religieuses si longtemps réprimées et, d'autre part, la priorité donnée dans notre travail d'enquête à la localisation des temples et à l'histoire de leur destin récent, ne nous ont pas permis d'approfondir notre connaissance des activités des associations de fidèles. Pour avoir accès à la mémoire des activités n'étant pas perçues comme séparées de la vie ordinaire et ne faisant pas l'objet d'une remémoration spéciale, il faudra entreprendre des enquêtes au long cours avec des témoins privilégiés, malheureusement de plus en plus rares.

Néanmoins, les matériaux réunis dessinent une première ébauche du paysage religieux formé par les temples de Pékin. Selon leur degré d'ouverture au public, et l'identité de ceux qui en assuraient l'entretien, on peut y distinguer plusieurs catégories. Pékin abritait d'abord des sanctuaires entièrement entretenus par la cour impériale et fermés au public, du fait qu'ils se situaient dans des espaces inaccessibles aux gens ordinaires ou que leur accès était interdit.

Les grandes fondations taoïstes, bouddhistes ou lamaïstes, abritant des dizaines, voire des centaines de religieux, et dans lesquelles se déroulait une activité rituelle continue - où l'étude et la récitation des textes occupaient l'essentiel du temps des moines -, forment une autre catégorie. L'espace de ces temples s'ouvrait aux fidèles à certains moments de l'année, qui correspondaient aux fêtes du calendrier liturgique ou d'anniversaire des divinités. Même dans ces occasions, les religieux y maintenaient des espaces privés où les laïcs, parfois considérés comme impurs, ne pénétraient pas. Ces temples de grandes dimensions étaient très peu nombreux.

Près de quatre-vingt-dix pour cent des temples étaient en fait de petite taille, formés d'un portique d'entrée, d'une grande salle sur cour, avec des bâtiments annexes, le tout entouré d'une enceinte. C'est dans ces fondations de taille modeste que l'on trouvait des temples occupés par un moine ou une nonne et ses disciples. Ceuxci étaient ouverts aux fidèles les $1^{\mathrm{er}}$ et $15^{\mathrm{e}}$ jours du mois, ou à tout autre moment sur demande faite au religieux, pour un rituel de guérison, un exorcisme ou l'organisation d'un enterrement. 
Les fondations gérées par une association de fidèles, une guilde ou un groupe sectaire formaient une autre catégorie. Ces temples n'étaient pas forcément ouverts aux fidèles ordinaires. Les membres des guildes professionnelles s'en servaient comme lieux de réunion, parfois y stockaient des marchandises ou y logeaient des hôtes de passage; d'autres temples étaient entretenus par les fonctionnaires d'une administration particulière, comme celui dédié à un geôlier, situé autrefois dans l'entrée de la prison du ministère de la Justice, qui nous est connu par une inscription de $1850^{30}$.

Il existait encore des temples privés ou familiaux réservés aux dévotions d'une famille particulière qui en avait la pleine propriété et entretenait éventuellement un religieux pour célébrer les cultes. Enfin une dernière catégorie, sans doute la plus répandue et la plus ordinaire, était celle des petits sanctuaires du dieu du Sol que l'on trouvait pratiquement à chaque coin de rue et qui, du fait de leur position stratégique, ont été très souvent remplacés par des toilettes publiques.

Aucune de ces catégories n'est complètement étanche, à l'exception, sous l'empire, de celle des temples réservés au service de l'empereur. Des groupes de fidèles laïcs, comme c'est encore le cas aujourd'hui, étaient souvent attachés à une grande fondation pour y mener leur vie religieuse. Ils assistaient régulièrement aux rituels et suivaient les cours donnés par des moines éminents sur des sutras. Lors des fêtes, ils préparaient les repas, surveillaient et nettoyaient les salles et les statues. Selon sa personnalité, le propriétaire d'un temple privé autorisait ou non le voisinage à venir prier dans le temple, du moins à venir y chercher conseil auprès du religieux. Les temples habités par un ou deux religieux, qui dépendaient pour leur entretien des services rituels rendus à la communauté, ont souvent abrité des associations de fidèles dont l'existence est attestée durant plusieurs décennies.

Partis à la recherche d'une organisation socioreligieuse particulière, nous avons été mis en présence d'une grande variété de situations où il est apparu que les temples de Pékin avaient été entretenus au cours des siècles par toutes les couches de la société sur des modes tantôt individuels, tantôt organisés, souvent ponctuels mais aussi durables. Cette courte présentation résume les principaux cas 
de figure, mais de longues études seront encore nécessaires pour saisir toutes les nuances du paysage religieux de la capitale.

EFEO-Pekin

Institute for History of Natural Science

Chaoyang mennei 137, 100010 Peking

P. R. of China

Tel : 00-86-10-64 006999 (office)

mariannebujard@yahoo.com.cn

efeo2002@yahoo.com.cn (office) 